\title{
On the Successful Delivery Probability of Full-Duplex-Enabled Mobile Edge Caching
}

\author{
Thang X. Vu ${ }^{\circledR}$, Lei Lei ${ }^{\circledR}$, Symeon Chatzinotas ${ }^{\circledR}$, Björn Ottersten ${ }^{\circledR}$, and Anh Vu Trinh
}

\begin{abstract}
Cache hit ratio (CHR) is a common metric to evaluate the performance of an edge caching system. While the CHR is efficient to measure throughput reduction gain, it fails to guarantee user quality of experience since the CHR does not capture the last-mile channel uncertainty. In this letter, we investigate a novel successful delivery probability (SDP) metric of full-duplex-enabled mobile edge caching (FD-MEC) systems, defined as a probability that the user receives the requested file before a tolerable delay. First, the average SDP is derived in a closed-form expression for arbitrary caching policy and network topology, taking into account the wireless fading distribution. Second, based on the derived SDP, a suboptimal cache placement is proposed to maximize the average SDP, which is analytically shown to surpass both the most popular and uniform caching policies. Finally, the numerical results are presented to verify the accuracy of our analysis and demonstrate the effectiveness of our proposed cache placement design.
\end{abstract}

Index Terms-Mobile edge caching, successful delivery probability, full duplex, cache placement, performance analysis.

\section{INTRODUCTION}

A MONG potential enabling technologies to tackle stringent latency and data-hungry challenges in future wireless networks, mobile edge caching (MEC) has received much attention. The basic premise of MEC is to prefetch content close to end users at the edge local storage. When the users request a content, it can be served directly by the edge node $(\mathrm{EN})$ without being transferred from the core network [1]. In order to efficiently exploit the cached content, joint design for content caching and physical layer has been considered recently. The main idea is to take into account the cached content at the ENs when designing the signal transmission to reduce costs on both access and backhaul links. Because some of the requested files are available in the EN's cache, proper design is required for content selection combined with broad/multi-cast transmission design to improve the system performance in terms of energy efficiency [2]-[4], throughputoutage tradeoff [5], and delivery time [6], [7]. The performance of MEC systems can be further improved by joint optimization of caching along with routing and resource allocation [8].

Manuscript received February 25, 2019; accepted March 28, 2019. Date of publication April 2, 2019; date of current version June 10, 2019. This research was supported by the FNR CORE ProCAST project (R-AGR-341510) and Vietnam National University, Hanoi, under project No. QG.18.39. The associate editor coordinating the review of this letter and approving it for publication was N. Tran. (Corresponding author: Thang X. Vu.)

T. X. Vu, L. Lei, S. Chatzinotas, and B. Ottersten are with the Interdisciplinary Centre for Security, Reliability and Trust, University of Luxembourg, L-1855 Luxembourg City, Luxembourg (e-mail: thang.vu@uni.lu; lei.lei@uni.lu; symeon.chatzinotas@uni.lu; bjorn.ottersten@uni.lu).

A. V. Trinh is with the Electronics and Telecommunications Department, VNU University of Engineering and Technology, Hanoi, Vietnam (e-mail: vuta@vnu.edu.vn).

Digital Object Identifier 10.1109/LCOMM.2019.2908847

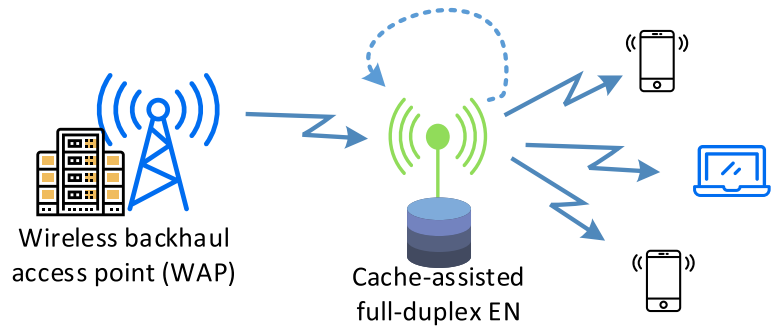

Fig. 1. Block diagram of the FD-MEC. Self-interference exists at the EN.

Meanwhile, full-duplex (FD) has shown great potential as a transmission technique for the next generation wireless networks. With recent advances in self-interference cancellation, FD can potentially double the spectral efficiency by allowing a node to transmit and receive signals on the same band simultaneously [9]. In [10], the authors show that cache-aided FD systems can provide cache hit enhancements compared with the half-duplex (HD) system via stochastic geometry analysis. The worst case normalized delivery time (NDT) in HetNets is studied in [11] with FD relaying nodes. Unfortunately, the results in [11] are based on an optimistic assumption that the self-interference can be fully mitigated. In practice, there always remains a residual self-interference (RSI) after the selfinterference cancellation.

In this letter, we investigate the impact of caching in fullduplex enabled MEC (FD-MEC) systems under a realistic model of the RSI. Firstly, we define and analyze the average successful delivery probability (SDP) of FD-MEC in a closedform expression by taking into consideration the distribution of all wireless fading channels. The derived SDP allows direct inspection of the caching policy and the key system parameters. Secondly, based on the derived SDP, we propose a cache placement design that maximizes the average SDP and surpasses both the most popular and uniform caching policies. Finally, numerical results verify the accuracy of our analysis and demonstrate the benefit of the proposed caching design over the benchmark references.

Notations: $\Gamma(k), \Gamma_{1}(x ; k, \theta), \Gamma_{2}(x ; k, \theta)$ denote the Gamma, lower incomplete, and upper incomplete Gamma functions, respectively. $\left(\begin{array}{l}n \\ k\end{array}\right)$ denotes the binomial coefficient.

\section{SySTEM MODEL}

We consider an FD-MEC in which the users request content via an FD EN, as depicted in Fig. 1. The EN connects to the core network via a wireless backhaul access point (WAP), e.g., macro base station. There is no direct WAP-user link, thus the 
users can only access data via the EN. The WAP is assumed to have full access to a library of $N$ content files, denoted by $\mathcal{D}=\left\{D_{1}, \ldots, D_{N}\right\}$. Without loss of generality, all contents are assumed to have equal size of $Q$ bits. To leverage the backhaul during peak-hours, the EN is equipped with a storage memory of $M Q$ bits, where $M<N$.

\section{A. Content Popularity and Caching Model}

We consider the most popular content popularity model, i.e., the Zipf distribution. The probability for file $D_{n}$ being requested is equal to $q_{n}=\frac{n^{-\alpha}}{\sum_{m=1}^{N} m^{-\alpha}}$, where $\alpha$ is the Zipf skewness factor.

We consider a generic caching policy $\boldsymbol{\mu}=\left[\mu_{1}, \ldots, \mu_{N}\right]$, where $0 \leq \mu_{n} \leq 1$ denotes portions of file $D_{n}$ prefetched at the $\mathrm{EN}$, where $\sum_{n=1}^{N} \mu_{n} \leq M$ to satisfy the memory constraint. The motivation behind the generic caching policy is that it allows the study of different caching strategies. For example, in the most popular caching we have $\mu_{n}=1,1 \leq$ $n \leq M$ and $\mu_{n}=0, \forall n>M$, and in the uniform caching, $\mu_{n}=M / N, \forall n$.

\section{B. Signal Transmission Model}

When a user requests a content, it sends the content index to the EN. The EN first checks the local cache. If (parts of) the requested content is available in the cache, it serves the user directly. Otherwise, the EN will receive the non-cached parts from the WAP via the wireless backhaul before serving the user. Let $d_{u}, \forall u$, denote the file index requested by user $u$. Denote $\breve{D}_{d_{u}}$ as the non-cached parts of file $D_{d_{u}}$ which will be sent from the WAP. Furthermore, denote $x_{1, u}$ and $x_{2, u}$ as the modulated signal of $\breve{D}_{d_{u}}$ and $D_{d_{u}}$, respectively. The EN employs the FastForward protocol [12] to send $x_{2, u}$ to the user while receiving $x_{1, u}$ from the WAP. In order to decode for $x_{1, u}$, the EN performs self-interference cancellation since $x_{2, u}$ is known.

We assume that the WAP is equipped with $L$ antennas, while the EN and users are equipped with a single antenna [10]. Denote $\overline{\boldsymbol{h}}=\sqrt{G_{1}} \boldsymbol{h} \in \mathbb{C}^{1 \times L}$ as the channel coefficients of the backhaul link, where $G_{1}$ is the path-loss degradation and $\boldsymbol{h}$ is the small-scale fading, whose elements are identically independently distributed (i.i.d.) complex Gaussian random variables with zero-mean and unit variance. Let $\bar{g}_{u}=$ $\sqrt{G_{2, u}} g_{u}$ denote the channel coefficient between the EN and user $u$, where $G_{2, u}$ is the pathloss from the EN to user $u$, and $g_{u}$ is the fading coefficient, which is complex Gaussian random variable with zero-mean and unit variance. The $U$ users are served by the time division multiple access (TDMA), in which each user is consecutively active in $\frac{T}{U}$, where $T$ is the coherence time. Therefore, there is no inter-user interference. However, since the EN operates in FD mode, there exists selfinterference at the EN. The effective achievable information rate (bit per second) on the backhaul and access links are respectively given as $R_{1, u}=\frac{B}{U} \log _{2}\left(1+\frac{P_{1} G_{1}|\boldsymbol{h} \boldsymbol{w}|^{2}}{I_{s i}+\sigma^{2}}\right), R_{2, u}=$ $\frac{B}{U} \log _{2}\left(1+\frac{P_{2} G_{2, u}\left|g_{u}\right|^{2}}{\sigma^{2}}\right)$, where $P_{1}, P_{2}$ are the transmit power at the WAP and the EN, respectively, $\boldsymbol{w}=\boldsymbol{h}^{H}$ is the maximum ratio transmit precoding vector for the backhaul link, where $(.)^{H}$ denotes the conjugate transpose, $I_{s i}$ is the residual self-interference power at the EN after interference cancellation.

Unlike some works which treat $I_{s i}$ as a constant noise floor, we employ a realistic model for the RSI, which models $I_{s i}$ as a Gamma distributed variable [13] with an integer shape parameter $K$ and a scale parameter $\theta$, i.e., $I_{s i} \sim \operatorname{Gamma}(K, \theta)$. The mean of $I_{s i}$ is $\bar{I}_{s i}=K \theta=$ $\eta P_{2}$, where $\eta$ is a constant depending on the cancellation efficiency [14].

\section{Successful Delivery Probability Analysis}

In this section, we derive a closed-form expression for the average SDP by taking into account the fading uncertainty at all channels. We assume that the users request different files, which occurs with high probability as the library size is usually much larger than the number of users. Since the analysis is similar for every user, we drop the user subscript $u$ for ease of presentation, i.e., $G_{2}, R_{1}, R_{2}$ are used instead of $G_{2, u}, R_{1, u}, R_{2, u}$.

Definition 1 (Successful Delivery Probability): The SDP of serving the requested file $D_{n}$ is defined as the probability that the user receives file $D_{n}$ before the maximum tolerable delay, defined as

$$
\Psi_{n}=\operatorname{Pr}\left\{t_{n} \leq \frac{Q}{\rho}\right\}
$$

where $t_{n}$ is the time to complete the transmission of file $D_{n}$ and $\rho$ is the minimum rate requirement.

Under the FastForward protocol [12], the end-to-end delivery time for transferring file $n$ is determined by the time for sending the non-cached parts $\breve{D}_{d_{u}}$ over the backhaul and the time for sending $D_{d_{u}}$ over the access link. Thus, $t_{n}=\max \left(\frac{\left(1-\mu_{n}\right) Q}{R_{1}}, \frac{Q}{R_{2}}\right)$.

Denote $\gamma_{1}=\frac{P_{1} G_{1}|\boldsymbol{h} \boldsymbol{w}|^{2}}{\sigma^{2}}, \gamma_{2}=\frac{P_{2} G_{2}|g|^{2}}{\sigma^{2}}$, and $\gamma_{s i}=\frac{I_{s i}}{\sigma^{2}}$, then the achievable rates can be rewritten as $R_{1}=\frac{B}{U} \log _{2}(1+$ $\left.\frac{\gamma_{1}}{\left(\gamma_{s i}+1\right)}\right)$ and $R_{2}=\frac{B}{U} \log _{2}\left(1+\gamma_{2}\right)$. Under Rayleigh fading channels, it is straightforward to verify that $\gamma_{1}$ follows a $\operatorname{Gamma}$ distribution $\operatorname{Gamma}\left(L, \bar{\gamma}_{1}\right)$ with $\bar{\gamma}_{1}=\frac{P_{1} G_{1}}{\sigma^{2}}$ and $\gamma_{2}$ follows an exponential distribution with a parameter $\bar{\gamma}_{2}=$ $\frac{P_{2} G_{2}}{\sigma^{2}}$. In addition, since $I_{s i} \sim \operatorname{Gamma}(K, \theta)$, then $\gamma_{s i} \sim$ $\operatorname{Gamma}\left(K, \bar{\gamma}_{s i}\right)$, where $\bar{\gamma}_{s i}=\frac{\theta}{\sigma^{2}}=\frac{\eta P_{2}}{\sigma^{2} K}$. The probability density function (PDF) of $\gamma_{1}, \gamma_{2}$ and $\gamma_{s i}$ are respectively given as

$$
\begin{aligned}
f_{\gamma_{1}}(x) & =\frac{x^{L-1} e^{-\frac{x}{\gamma_{1}}}}{\bar{\gamma}_{1}^{L} \Gamma(L)}, \quad f_{\gamma_{2}}(y)=\frac{e^{-\frac{y}{\gamma_{2}}}}{\bar{\gamma}_{2}}, \\
f_{\gamma_{s i}}(z) & =\frac{z^{K-1} e^{-\frac{z}{\bar{\gamma}_{s i}}}}{\bar{\gamma}_{s i}^{K} \Gamma(K)} .
\end{aligned}
$$

Our main result is given in the theorem below.

Theorem 1: The average SDP of an FD-MEC system under a caching policy $\boldsymbol{\mu}$ and a rate requirement $\rho$ is given as

$$
\operatorname{SDP}(\boldsymbol{\mu}, \rho)=\sum_{n=1}^{N} q_{n} \Psi_{n}(\boldsymbol{\mu}, \rho)
$$


where $\Psi_{n}(\boldsymbol{\mu}, \rho)=\left(1-F_{\gamma_{b h}}\left(e^{\frac{\left(1-\mu_{n}\right) \bar{\rho} U}{B}}-1\right)\right) \exp \left(\frac{1-e^{\frac{\bar{\rho} U}{B}}}{\bar{\gamma}_{2}}\right)$, $\bar{\rho}=\rho \log (2), q_{n}$ is the popularity of file $D_{n}$, and

$$
\begin{aligned}
F_{\gamma_{b h}}(x)= & \frac{e^{\frac{1}{\bar{\gamma}_{s i}}} x^{L}}{\bar{\gamma}_{1}^{L} \Gamma(L)} \sum_{p=0}^{K-1} \frac{1}{\bar{\gamma}_{s i}^{p}} \sum_{q=0}^{p}\left[\frac{(-1)^{q}}{q !(p-q) !}\left(\frac{x}{\bar{\gamma}_{1}}+\frac{1}{\bar{\gamma}_{s i}}\right)^{q-p-L}\right. \\
& \left.\times \Gamma_{2}\left(\frac{x}{\bar{\gamma}_{1}}+\frac{1}{\bar{\gamma}_{s i}} ; L+p-q, 1\right) \Gamma(L+p-q)\right] \\
& +\Gamma_{1}\left(\frac{x}{\bar{\gamma}_{1}} ; L, 1\right) .
\end{aligned}
$$
have

Proof: Substituting $t_{n}=\max \left(\frac{\left(1-\mu_{n}\right) Q}{R_{1}}, \frac{Q}{R_{2}}\right)$ into (1) we

$$
\begin{aligned}
\Psi_{n}(\boldsymbol{\mu}, \rho)= & \operatorname{Pr}\left\{\max \left(\left(1-\mu_{n}\right) Q / R_{1}, Q / R_{2}\right) \leq Q / \rho\right\} \\
= & \operatorname{Pr}\left\{\frac{\left(1-\mu_{n}\right) Q U}{B \log _{2}\left(1+\frac{\gamma_{1}}{\gamma_{s i}+1}\right)} \leq \frac{Q}{\rho}\right\} \\
& \times \operatorname{Pr}\left\{\frac{Q U}{B \log _{2}\left(1+\gamma_{2}\right)} \leq \frac{Q}{\rho}\right\} \\
= & A_{1} \times A_{2},
\end{aligned}
$$

where $A_{1} \triangleq \operatorname{Pr}\left\{\log \left(1+\frac{\gamma_{1}}{\gamma_{s i}+1}\right) \geq \frac{\bar{\rho}\left(1-\mu_{n}\right) U}{B}\right\}$ and $A_{2} \triangleq$ $\operatorname{Pr}\left\{\log \left(1+\gamma_{2}\right) \geq \frac{\bar{\rho} U}{B}\right\}$, with $\bar{\rho} \triangleq \rho \log (2)$.

Since $\gamma_{2}$ follows an exponential distribution with parameter $\bar{\gamma}_{2}=P_{2} G_{2} / \sigma^{2}$, we directly obtain $A_{2}=\exp \left(-\frac{e^{\frac{\rho}{B}}-1}{\bar{\gamma}_{2}}\right)$. On the other hand, the computation of $A_{1}$ is challenging since both $\gamma_{1}$ and $\gamma_{s i}$ are Gamma distributed random variables.

In order to overcome this difficulty, we introduce an intermediate variable $\gamma_{b h}=\frac{\gamma_{1}}{\gamma_{s i}+1}$, which can be seen as the instantaneous signal-to-noise-plus-interference ratio on the backhaul link, that is also a random variable. Therefore, we have

$$
A_{1}=\operatorname{Pr}\left\{\gamma_{b h} \geq e^{\frac{\left(1-\mu_{n}\right) \bar{\rho} U}{B}}-1\right\}=1-F_{\gamma_{b h}}\left(e^{\frac{\left(1-\mu_{n}\right) \bar{\rho} U}{B}}-1\right) \text {, }
$$

where $F_{\gamma_{b h}}(x)$ is the CDF of $\gamma_{b h}$. By using Lemma 2 shown in the Appendix, we can immediately compute $A_{1}$. Substituting $A_{1}, A_{2}$ into (3) we have Theorem 1 proved.

Theorem 1 provides a closed-form expression for the average SDP of the considered FD-MEC under arbitrary caching policy and network topology. This general result allows direct inspection of the FD-MEC in some special scenarios.

Proposition 1: The average SDP of the FD-MEC under the uniform caching policy, $\mathrm{SDP}^{U n i}$, and under the most popular caching, $\mathrm{SDP}^{P o p}$, are respectively given as follows:

$$
\begin{aligned}
& \operatorname{SDP}^{U n i}=e^{-\frac{\tilde{\rho}}{\bar{\gamma}_{2}}}\left(1-F_{\gamma_{b h}}(\bar{\mu})\right), \\
& \mathrm{SDP}^{\text {Pop }}=e^{-\frac{\tilde{\rho}}{\gamma_{2}}}\left(Q_{M}+\left(1-Q_{M}\right)\left(1-F_{\gamma_{b h}}(\tilde{\rho})\right)\right),
\end{aligned}
$$

where $F_{\gamma_{b h}}(x)$ is given in Lemma $2, \tilde{\rho}=e^{\bar{\rho} U / B}-1, \bar{\mu}=$ $e^{\left(1-\frac{M}{N}\right) \frac{\bar{\rho} U}{B}}-1$, and $Q_{M} \triangleq \sum_{n=1}^{M} q_{n}$.

The results in Proposition 1 can be immediately obtained from Theorem 1 by using the uniform and most popular caching vectors $\boldsymbol{\mu}_{\mathrm{UNI}}=\left[\frac{M}{N}, \ldots, \frac{M}{N}\right]$ and $\boldsymbol{\mu}_{\mathrm{POP}}=[\underbrace{1, \ldots, 1}_{\times M}, 0, \ldots, 0]$.

Proposition 2: Consider the FD-MEC with a single-antenna at the WAP and an exponential distribution model of the
TABLE I

Algorithm to SOLVE (9)

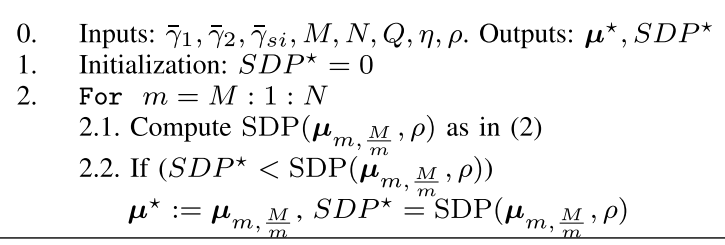

RSI, i.e., $L=1$ and $K=1$. Denote $\bar{\mu}_{n}=e^{\frac{\left(1-\mu_{n}\right) \bar{\rho} U}{B}}-1$, the average SDP in this case is given as

$$
\mathrm{SDP}^{\text {single }}=\sum_{n=1}^{N} \frac{q_{n} \bar{\gamma}_{1}}{\bar{\gamma}_{s i} \bar{\mu}_{n}+\bar{\gamma}_{1}} e^{-\frac{\bar{\mu}_{n}}{\bar{\gamma}_{1}}-\frac{\tilde{\rho}}{\gamma_{2}}} .
$$

\section{Cache Placement Design Maximizing the SDP}

It is well known that the most popular caching policy is optimal in terms of CHR and throughput reduction. However, this might not be the best choice for the SDP since this policy does not take into account the last-mile channel quality [3]. In this section, we optimize the prefetched content in order to maximize the average SDP. In particular, we optimize the cached portions $\mu_{n}$ of the $n$-th file subject to the memory constraint. The optimization problem is formulated as follows:

$$
\underset{\left\{\mu_{n}: 0 \leq \mu_{n} \leq 1\right\}_{n=1}^{N}}{\operatorname{maximize}} \operatorname{SDP}(\boldsymbol{\mu}, \rho), \quad \text { s.t. } \sum_{n=1}^{N} \mu_{n} \leq M
$$

where $\boldsymbol{\mu} \triangleq\left[\mu_{1}, \mu_{2}, \ldots, \mu_{N}\right]$ is a caching vector and $\operatorname{SDP}(\boldsymbol{\mu}, \rho)$ is computed as in Theorem 1 .

Finding the optimal solution of problem (8) is in general very challenging because the function $\operatorname{SDP}(\boldsymbol{\mu}, \rho)$ is neither convex nor concave in $\boldsymbol{\mu}$. Instead, we target a suboptimal solution by confining the original $N$-dimensional search space into a linear one. Specifically, we consider that the EN prefetches the same portions of all cached files, i.e., $\mu_{n}=\mu$ if file $D_{n}$ is cached, and $\mu_{n}=0$ otherwise. This way, the caching policy can be described via the vector $\boldsymbol{\mu}_{m, \mu} \triangleq[\mu, \ldots, \mu, 0, \ldots, 0]$, (first $m$ elements equal to $\mu$ ), where $M \leq m \leq N$.

Then the resulted problem can be formulated as

$\underset{m \in \mathbb{Z}^{+}, \mu>0}{\operatorname{maximize}} \operatorname{SDP}\left(\boldsymbol{\mu}_{m, \mu}, \rho\right), \quad$ s.t. $M \leq m \leq N ; m \mu \leq M$.

Because the function $\operatorname{SDP}\left(\boldsymbol{\mu}_{m, \mu}, \rho\right)$ is strictly increasing over $\mu$ for a given $m$, the optimal fractional cache is $\mu^{\star}=\frac{M}{m}$. As a result, problem (9) reduces to a search of $\operatorname{SDP}\left(\boldsymbol{\mu}_{m, \underline{M}}, \rho\right)$ over $N-M+1$ possibilities of $m$. The algorithm to solve (9) is described in Table 1.

Lemma 1: The caching solution of problem (9) in the considered FD-MEC system always achieves a higher SDP than the most popular and uniform caching strategies.

\section{NUMERICAL RESULTS}

This section presents numerical results to demonstrate the accuracy of our analysis and the effectiveness of the proposed 
(a)

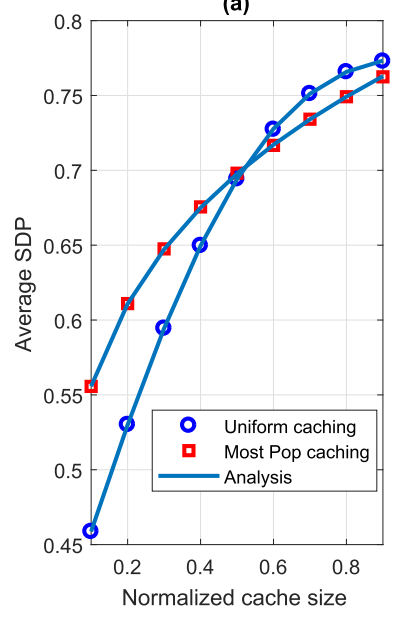

(b)

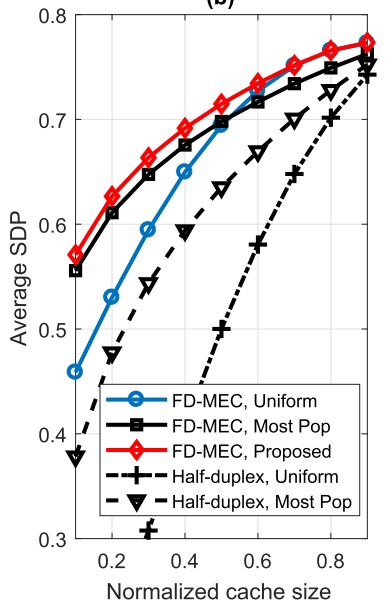

Fig. 2. (a) - Accuracy of our analysis. (b) - SDP comparison of the proposed cache placement with references. Simulation parameters: $P_{1}=20 \mathrm{~W}$, $P_{2}=1 \mathrm{~W}, \sigma^{2}=-120 \mathrm{dBm}$, and $\rho=10 \mathrm{Mbps}$.

caching policy. The content requests follow the Zipf distribution with a skewness factor $\alpha=0.8$. The EN is 200 meters far from the WAP. The users are randomly located between 50 to 75 meters from the EN. We employ the B5a pathloss model [15], which results in $G_{1}=-90 \mathrm{~dB}$ and $-80 \mathrm{~dB} \leq$ $G_{2} \leq-76 \mathrm{~dB}$. In addition, $Q=100 \mathrm{Mb}, B=20 \mathrm{MHz}, U=4$ users, $L=2, K=2, N=100$ files, and $\eta=-83 \mathrm{~dB}$.

Fig. 2a shows the average SDP of the FD-MEC as a function of the normalized cache size $\frac{M}{N}$. It is shown that the analysis matches very well with the simulations for both the most popular and uniform caching policies, which confirms the accuracy of our analysis. Interestingly, the uniform caching achieves a smaller SDP when the cache size is limited, but surpasses the most popular policy for larger cache sizes, which is in contrast to the common understanding that the most popular policy is always better than the uniform caching.

Fig. 2b compares the SDP of the proposed caching strategy with the two benchmark policies. In addition, we also include the SDP of the half-duplex system, in which the transmission on the backhaul and access links occur in two consecutive time slots. It is shown that the proposed caching strategy surpasses all other schemes.

\section{CONCLUSION}

In this letter, we have investigated the performance of full-duplex enabled mobile edge caching systems in terms of successful delivery probability, which takes into account both the cached content and the last-mile channel quality. We analyzed the average SDP in a closed-form expression, which allows direct examination of arbitrary caching policy and other system key parameters. In addition, we proposed a suboptimal cache placement that outperforms both the most popular and uniform caching policies in the considered singlecell system. The outcome of this work suggests a new cache placement design to fully exploit the potentials of full-duplex in general MEC systems. In such cases, the cache placement should take into account possible inter-cell interference on top

of the self-interference. A promising topic is to investigate the impact of massive MIMO-enabled backhaul on the multi-cell FD-MEC context.

\section{APPENDIX}

Lemma 2: The CDF of the SINR $\gamma_{b h}$ can be computed as

$$
\begin{aligned}
& F_{\gamma_{b h}}(x) \\
& =\frac{e^{\frac{1}{\bar{\gamma}_{s i}}}}{\bar{\gamma}_{1}^{L} \Gamma(L)} \sum_{p=0}^{K-1} \frac{x^{L}}{\bar{\gamma}_{s i}^{p}} \sum_{q=0}^{p} \frac{(-1)^{q}}{q !(p-q) !}\left(\frac{x}{\bar{\gamma}_{1}}+\frac{1}{\bar{\gamma}_{s i}}\right)^{q-p-L} \\
& \quad \times \Gamma_{2}\left(\frac{x}{\bar{\gamma}_{1}}+\frac{1}{\bar{\gamma}_{s i}} ; L+p-q, 1\right)+\Gamma_{1}\left(\frac{x}{\bar{\gamma}_{1}} ; L, 1\right) .
\end{aligned}
$$

Proof: The CDF of $\gamma_{s i}=\frac{\gamma_{1}}{\gamma_{s i}+1}$ is computed as follows:

$$
\begin{aligned}
F_{e q}(t) & =\operatorname{Pr}\left\{\frac{\gamma_{1}}{\gamma_{s i}+1} \leq t\right\}=\operatorname{Pr}\left\{\gamma_{1} \leq t\left(\gamma_{s i}+1\right)\right\} \\
& =\operatorname{Pr}\left\{\gamma_{1} \leq t\left(\gamma_{s i}+1\right) \mid \gamma_{1}\right\} \operatorname{Pr}\left\{\gamma_{1}\right\} \\
& \stackrel{(a)}{=} \operatorname{Pr}\left\{\gamma_{1} \leq t\right\}+\operatorname{Pr}\left\{\gamma_{s i} \geq \frac{\gamma_{1}}{t}-1 \mid \gamma_{1}\right\} \operatorname{Pr}\left\{\gamma_{1} \geq t\right\} \\
& \stackrel{(b)}{=} \Gamma_{1}\left(t ; L, \bar{\gamma}_{1}\right)+I_{2}(t),
\end{aligned}
$$

where $(a)$ is because $\gamma_{s i}>\frac{\gamma_{1}}{t}-1, \forall \gamma_{1}<t,(b)$ is obtained since $\gamma_{1}$ follows a $\operatorname{Gamma}$ distribution $\operatorname{Gamma}\left(L, \bar{\gamma}_{1}\right)$, and $I_{2}(t)=\int_{x=t}^{+\infty}\left[\int_{y=x / t-1}^{+\infty} f_{\gamma_{s i}}(y) d y\right] f_{\gamma_{1}}(x) d x$.

Since $\gamma_{s i}$ is also a Gamma random variable with a shape $K$ and a scale $\bar{\gamma}_{s i}$, we can obtain:

$$
\begin{aligned}
I_{2}(t) & =\int_{x=t}^{+\infty}\left(\int_{y=x / t-1}^{+\infty} \frac{y^{K-1} e^{-\frac{y}{\bar{\gamma}_{s i}}}}{\bar{\gamma}_{s i}^{K} \Gamma(K)} d y\right) \frac{x^{L-1} e^{-\frac{x}{\bar{\gamma}_{1}}}}{\bar{\gamma}_{1}^{L} \Gamma(L)} d x \\
& =\int_{x=t}^{+\infty} \Gamma_{2}\left(\frac{x}{t}-1 ; K, \bar{\gamma}_{s i}\right) \frac{x^{L-1} e^{-\frac{x}{\gamma_{1}}}}{\bar{\gamma}_{1}^{L} \Gamma(L)} d x
\end{aligned}
$$

Because $K$ is an integer, we can decompose $\Gamma_{2}\left(x ; m, \bar{\gamma}_{s i}\right)$ into a summation form as follows:

$$
\Gamma_{2}\left(x ; K, \bar{\gamma}_{s i}\right)=\sum_{p=0}^{K-1} \frac{1}{p !}\left(\frac{x}{\bar{\gamma}_{s i}}\right)^{p} e^{-\frac{x}{\bar{\gamma}_{s i}}}
$$

Substituting (13) into (12) we obtain:

$$
\begin{aligned}
I_{2}( & () \\
= & \int_{x=t}^{+\infty} \sum_{p=0}^{K-1} \frac{e^{\frac{1}{\bar{\gamma}_{s i}}}}{p !\left(\bar{\gamma}_{s i} t\right)^{p}}(x-t)^{p} \frac{x^{L-1} e^{-x\left(\frac{1}{\bar{\gamma}_{1}}+\frac{1}{\bar{\gamma}_{s i} t}\right)}}{\bar{\gamma}_{1}^{L} \Gamma(L)} d x \\
= & \frac{e^{\frac{1}{\bar{\gamma}_{s i}}}}{\bar{\gamma}_{1}^{L} \Gamma(L)} \int_{x=t}^{+\infty} \sum_{p=0}^{K-1} \frac{1}{p !\left(\bar{\gamma}_{s i} t\right)^{p}} \\
& \times \sum_{q=0}^{p}(-1)^{q}\left(\begin{array}{l}
p \\
q
\end{array}\right) x^{p-q} t^{q} x^{L-1} e^{-x\left(\frac{1}{\bar{\gamma}_{1}}+\frac{1}{\bar{\gamma}_{s i} t}\right)} d x \\
= & \frac{e^{\frac{1}{\bar{\gamma}_{s i}}}}{\bar{\gamma}_{1}^{L} \Gamma(L)} \sum_{p=0}^{K-1} \frac{1}{p !\left(\bar{\gamma}_{s i} t\right)^{p}} \sum_{q=0}^{p}(-1)^{q}\left(\begin{array}{l}
p \\
q
\end{array}\right) t^{q} \\
& \times \int_{x=t}^{+\infty} x^{L+p-q-1} e^{-x\left(\frac{1}{\bar{\gamma}_{1}}+\frac{1}{\gamma_{s i} t}\right)} d x \\
= & \frac{e^{\frac{1}{\bar{\gamma}_{s i}}}}{\bar{\gamma}_{1}^{L} \Gamma(L)} \sum_{p=0}^{K-1} \frac{t^{L}}{\bar{\gamma}_{s i}^{p}} \sum_{q=0}^{p} \frac{(-1)^{q}}{q !(p-q) !}\left(\frac{t}{\bar{\gamma}_{1}}+\frac{1}{\bar{\gamma}_{s i}}\right)^{q-p-L} \\
& \times \Gamma_{2}\left(\frac{t}{\bar{\gamma}_{1}}+\frac{1}{\bar{\gamma}_{s i}} ; L+p-q, 1\right) \Gamma(L+p-q) .
\end{aligned}
$$

Substituting (16) into (11) we obtain Lemma 2. 


\section{REFERENCES}

[1] S. Borst, V. Gupta, and A. Walid, "Distributed caching algorithms for content distribution networks," in Proc. IEEE Int. Conf. Comput. Commun., Mar. 2010, pp. 1-9.

[2] F. Gabry, V. Bioglio, and I. Land, "On energy-efficient edge caching in heterogeneous networks," IEEE J. Sel. Areas Commun., vol. 34, no. 12, pp. 3288-3298, Dec. 2016.

[3] T. X. Vu, S. Chatzinotas, and B. Ottersten, "Edge-caching wireless networks: Performance analysis and optimization," IEEE Trans. Wireless Commun., vol. 17, no. 4, pp. 2827-2839, Apr. 2018.

[4] T. X. Vu, S. Chatzinotas, B. Ottersten, and T. Q. Duong, "Energy minimization for cache-assisted content delivery networks with wireless backhaul," IEEE Wireless Commun. Lett., vol. 7, no. 3, pp. 332-335, Jun. 2018.

[5] M. Ji, G. Caire, and A. F. Molisch, "Wireless device-to-device caching networks: Basic principles and system performance," IEEE J. Sel. Areas Commun., vol. 34, no. 1, pp. 176-189, Jan. 2016.

[6] F. Xu, M. Tao, and K. Liu, "Fundamental tradeoff between storage and latency in cache-aided wireless interference networks," IEEE Trans. Inf. Theory, vol. 63, no. 11, pp. 7464-7491, Nov. 2017.

[7] T. X. Vu, L. Lei, S. Vuppala, A. Kalantari, S. Chatzinotas, and B. Ottersten, "Latency minimization for content delivery networks with wireless edge caching," in Proc. IEEE Int. Conf. Commun., Kansas City, MO, USA, May 2018, pp. 1-6.
[8] A. Khreishah, J. Chakareski, and A. Gharaibeh, "Joint caching, routing, and channel assignment for collaborative small-cell cellular networks," IEEE J. Sel. Areas Commun., vol. 34, no. 8, pp. 2275-2284, Aug. 2016.

[9] A. Sabharwal, P. Schniter, D. Guo, D. W. Bliss, S. Rangarajan, and R. Wichman, "In-band full-duplex wireless: Challenges and opportunities," IEEE J. Sel. Areas Commun., vol. 32, no. 9, pp. 1637-1652, Sep. 2014.

[10] M. Maso, I. Atzeni, I. Ghamnia, E. Baçtuğ, and M. Debbah, "Cacheaided full-duplex small cells," in Proc. 15th Int. Symp. Modeling Opt. Mobile, Ad Hoc, Wireless Netw. (WiOpt), May 2017, pp. 1-6.

[11] J. Kakar, A. Alameer, A. Chaaban, A. Sezgin, and A. Paulraj, "Delivery time minimization in edge caching: Synergistic benefits of subspace alignment and zero forcing," in Proc. IEEE Int. Conf. Commun., May 2018, pp. 1-6.

[12] D. Bharadia and S. Katti, "Fastforward: Fast and constructive full duplex relays," in Proc. ACM Conf. SIGCOMM, 2014, pp. 199-210.

[13] N. H. Mahmood, I. S. Ansari, G. Berardinelli, P. Mogensen, and K. A. Qaraqe, "Analysing self interference cancellation in full duplex radios," in Proc. IEEE Wireless Commun. Netw. Conf., Apr. 2016, pp. 1-6.

[14] D. Bharadia and S. Katti, "Full duplex MIMO radios," in Proc. 11th USENIX Conf. Netw. Sys. Design Implement. (NSDI), Berkeley, CA, USA: USENIX Association, 2014, pp. 359-372.

[15] P. Kyosti et al., "WINNER II channel models," IST, Tech. Rep. D1.1.2 V1.2, 2007. 